\title{
Automated generation of context-specific Gene Regulatory Networks with a weighted approach in D. melanogaster
}

\author{
Leandro Murgas ${ }^{1, \dagger}$ \\ Sebastian Contreras-Riquelme $e^{1,2, \dagger}$ \\ J. Eduardo Martínez ${ }^{1,3}$, \\ Camilo Villaman ${ }^{1}$ \\ Rodrigo Santibáñez ${ }^{1}$, \\ and Alberto J.M. Martin ${ }^{1 *}$ \\ Santiago, 8580745, Chile, \\ $\dagger$ both authors contributed equally \\ *alberto.martin@umayor.cl
}

${ }^{1}$ Centro de Genónica y Bioinformática, Facultad de Ciencias, Universidad Mayor,

${ }^{2}$ Facultad de Ciencias de la Vida, Universidad Andres Bello, Santiago, 8370146, Chile,

${ }^{3}$ Centro de Modelamiento Molecular, Biofísica y Bioinformática - CM2B2, Facultad de Ciencias Químicas y Farmaceuticas, Universidad de Chile, Santiago, 8380492, Chile, and

\begin{abstract}
Motivation: The regulation of gene expression is a key factor in the development and maintenance of life in all organisms. This process is carried out mainly through the action of transcription factors (TFs), although other actors such as ncRNAs are involved. In this work, we propose a new method to construct Gene Regulatory Networks (GRNs) depicting regulatory events in a certain context for Drosophila melanogaster. Our approach is based on known relationships between epigenetics and the activity of transcription factors.

Results: We developed method, Tool for Weighted Epigenomic Networks in D. melanogaster (Fly T-WEoN), which generates GRNs starting from a reference network that contains all known gene regulations in the fly. Regulations that are unlikely taking place are removed by applying a series of knowledge-based filters. Each of these filters is implemented as an independent module that considers a type of experimental evidence, including DNA methylation, chromatin accessibility, histone modifications, and gene expression. Fly T-WEoN is based on heuristic rules that reflect current knowledge on gene regulation in D. melanogaster obtained from literature. Experimental data files can be generated with several standard procedures and used solely when and if available.Fly T-WEoN is available as a Cytoscape application that permits integration with other tools, and facilitates downstream network analysis. In this work, we first demonstrate the reliability of our method to then provide a relevant application case of our tool: early development of D. melanogaster.
\end{abstract}

Availability: Fly T-WEoN, together with its step-by-step guide is available at https://weon. readthedocs.io

Contact: alberto.martin@umayor.cl

Keywords: Systems biology, Gene regulation, Data integration, Condition-specific networks, Cytoscape 


\section{Introduction}

The regulation of gene expression is indispensable for adaptation to ever changing contexts and every aspect involved in sustaining life. Gene regulation is mainly carried out by highly specialized proteins, among which, Transcription Factors (TFs) are generally accepted as the key actors [22]. Canonically speaking, the regulation of gene expression works through the binding of TFs to certain sites in the chromatin, TF Binding Sites (TFBSs), and TFs recognize specific DNA patterns called TF Binding Motifs (TFBMs). These sites are usually unique for each TF, and they are commonly located around the promoter of TF-target genes upstream of their transcription start site. Whereas proximal upstream location of TFBS are easily related to the regulation of specific genes $[25,17]$, to determine which genes are controlled by each TF binding to enhancer regions have shown a greater difficulty [16, 21, 40]. Moreover, gene expression can be defined as the process by which the final products encoded by genes are generated, and thus, their regulation can also include control of translation and RNA degradation. In this way, several other non-TF regulatory elements are involved in the regulation of gene expression. For example, miRNAs and other ncRNAs are known to act during translation by binding to other RNAs $[8,3]$, while histone modifiers attach or remove post-translational modifications to control the positions of the chromatin that are available to be occupied by TFs.

Several epigenetic marks, including histone modifications [4] and DNA methylation [30], have been related to active and inactive states of chromatin [5, 15], therefore, influencing the ability of TFs to regulate gene expression. In this way, combinations of epigenetic marks have been related to a specific effect on TF-binding and gene expression, coining an epigenetic code that is still not properly understood $[4,2]$. Even so, there are some generally accepted facts on the relationship between TF-binding and epigenetic marks that have made possible to grasp a general tendency [33]. Nonetheless, chromatin structure and epigenetic marks change dynamically in a context-specific manner, and those changes have been subject of both static and dynamic modeling to predict gene expression [36].

Despite the relationship between epigenetic marks and gene regulation, the determination of the chromatin state for each TFBS remains experimentally difficult and expensive, while computational inference from limited experimental evidence is common in the literature. For instance, CENTIPEDE [31] is probably one of the first computational methods aiming to decipher which TFBS are actually bound at certain experimental condition instead of just defining TFBS from databases such as JASPAR [19]. CENTIPEDE makes use of DNase-seq data in an unsupervised learning algorithm to infer which TFBS are in an open active state and can compare its results with experimental data. Currently, computational analysis has at its disposal several tools to process experimental data related to gene regulation from which choosing is not an easy task. Nonetheless, some collaborative projects employ reliable pipelines, e.g. the TCGA workflow [37] or the ENCODE data processing pipelines (https://github.com/ENCODE-DCC). Often, those computational tools do not provide an intuitive interface, relying entirely on command-line instructions and/or do not report figures to interpret results from such data. For example, CENTIPEDE is a $\mathrm{R}$ package and, therefore, requires a minimum coding expertise. Moreover, there are other tools such as Anchor, a python package [23], Mocap, a python and R hybrid package, [10], and TEPIC, a C++ program [34]. All these methods aim to determine DNA occupancy by TFs, but require expertise from users in compiling, installing dependencies, coding, and the use of the command-line interfaces.

To overcome these difficulties, we created an efficient and easy to use method, Tool Weighted EpigenOmic Network (Fly T-WEoN), that is able to generate Drosophila melanogaster context-specific Gene Regulatory Networks (GRNs). This method employs a series of filters, that once applied to a reference network, remove TF-gene regulations that are unlikely taking place according to current knowledge on the relationship between epigenetic and TFBS activation. Specificity on resulting networks is provided by the time and context for which the omic data employed by each filter was generated. Our tool is available as a Cytoscape application that provides a user-friendly and intuitive interface where researchers easily introduce their data processed with standard protocols to generate context-specific GRNs.

\section{Methods}

\subsection{Construction of a Reference GRN}

A Reference GRN is a network that contains all known regulatory interactions between gene products and genes, regardless of developmental stage, environment or cell type in an organism. To create a reference network for D. melanogaster, we combined TFBS information from the ENCODE data repository [13] and FlyBase [18] to then infer regulatory relationships based on distance of TFBSs to the Transcription Start 


\begin{tabular}{ccc}
\hline Modification & Effect & References \\
\hline H3K27me3 & - & {$[7,42]$} \\
H3K36me2 & + & {$[4]$} \\
H3K36me3 & + & {$[4,15,20,7]$} \\
H3K4me1 & + & {$[4]$} \\
H3K4me2 & + & {$[4,7]$} \\
H3K4me3 & + & {$[4,15,7]$} \\
H3K79me2 & + & {$[35]$} \\
H3K9ac & + & {$[42,7]$} \\
H3K9me2 & - & {$[41,15,32]$} \\
H3K9me3 & - & {$[32,42]$} \\
H3S10ph & + & {$[7]$} \\
H4K16ac & + & {$[4]$} \\
H4K20me3 & - & {$[4]$} \\
\hline
\end{tabular}

Table 1: Histone modifications considered in Fly T-WEoN and their default effect. Effect of the histone marks on the binding of TFs to chromatin. "+" symbols indicate marks that allow TF binding and "-" indicate non-active TFBSs.

Site (TSS) of each gene in the genome of the fruit fly version 6.32 (see Supp. File NetsInfo for details). To determine whether a TF regulates a gene, we chose distance thresholds between TFBSs and the TSS of each gene, so if the TFBSs falls within this distance, we assumed it regulates the respective gene. We created three reference networks with different distance thresholds, 1500, 2000, and 5000 nucleotides inspired by other approaches [6]. In the case of miRNA, genetic relationships based on experimentally determined targets from miRecords [39] and miRTarBase [11] were also retrieved and incorporated into the reference networks.

\section{$2.2 \quad$ Filtering the reference network}

In order to determine which regulatory relationships are taking place in any experimental context of interest, we defined several filters, each relying on a different type of experimental data as input. The filtering process was implemented in PERL and is the backend software of the Cytoscape [12] application developed to provide a tool with a user-friendly interface. The filtering procedure generates a time and tissue-specific GRN depending on the experimental condition in which experimental data used was generated.

Our method considers experimental information following this order for each TFBS: chromatin accessibility (DNase-seq), methylation of the DNA, histones modifications around the TFBS, the expression of each TF with known TFBSs in the reference network and miRNA quantification (see Fig. 1). First, if there is a positive signal in the TFBSs for DNA methylation, Fly T-WEoN assumes that TFs cannot bind its TFBS and the filter removes the regulation accordingly. Second, if chromatin accessibility data, e.g., DNase-seq, shows a positive signal within the chosen distance threshold used to assign a TF to the regulation of a gene, this indicates that a TF can bind the corresponding region and therefore, the edge is not removed. The next filter considers if the chromatin is in open or closed state based on histone marks experimentally associated to this process. For example, trimethylation of the Histone H3 Lys27 [42, 7] or trimethylation of the Histone H4 Lys20 [41, 4, 20] for a inactive chromatin. The effect of the histone marks considered by default in the histone marks filter are described in Table 1, and sequencing reports in BED format were used as provided in ENCODE and FlyBase. Finally, the last filter considers if the gene coding a regulator (TF or miRNA) is expressed, regulations emerging from that node are kept in the final network.

\subsubsection{Scoring edges}

Fly T-WEoN assigns weights to edges in the resulting network. The weight of each edge is calculated by adding a score of one for each filter that the edge passes. By default, edges have no weight, so a weight of one means the edge passed only the expression filter, a weight of two means it passed an additional filter such as a histone mark, and a weight of three indicates that the edge passed the expression filter, and for example, two different histone modifications indicated its binding site was active. 


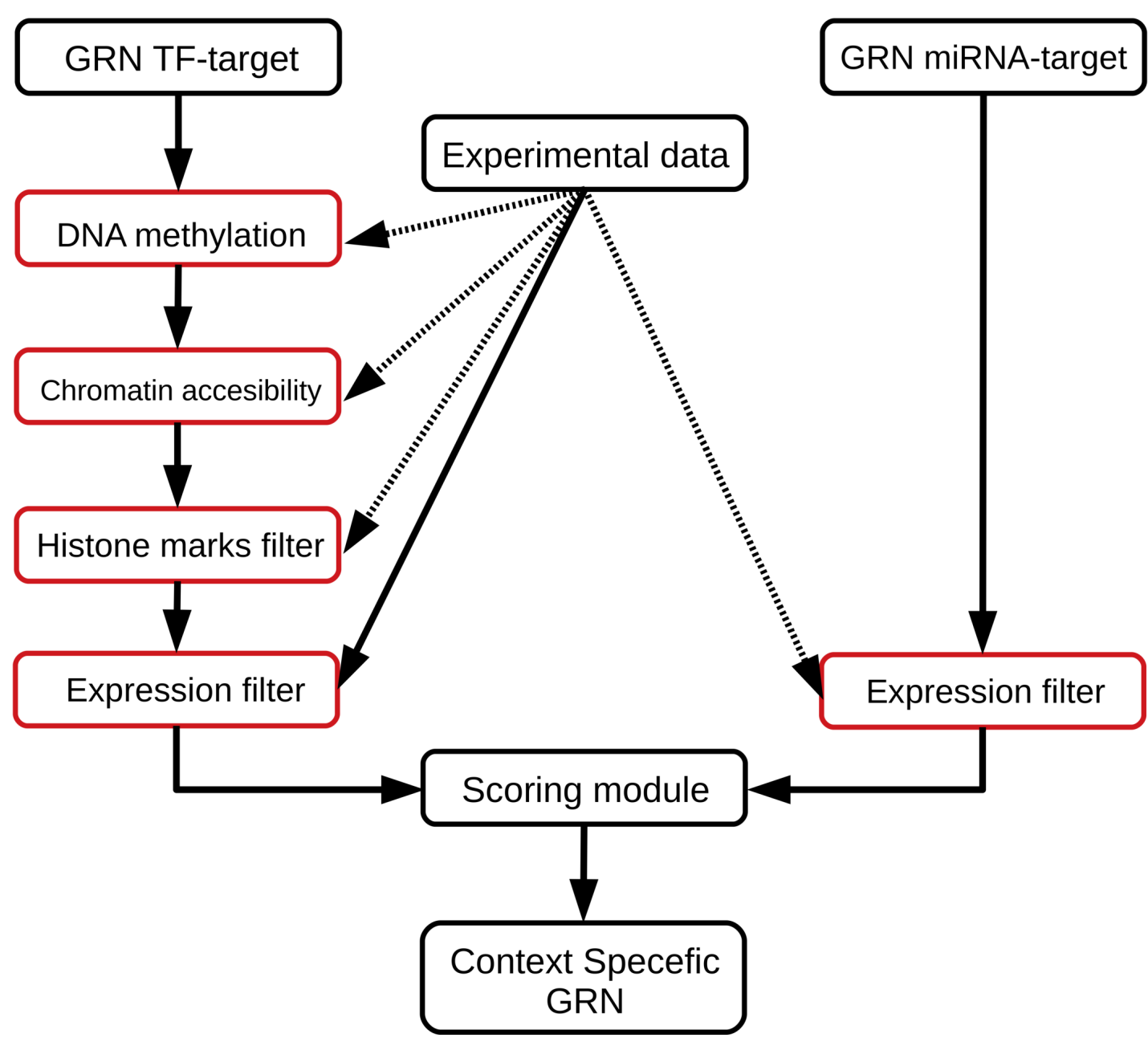

Figure 1: Flowchart describing Fly T-WEoN. The TF-gene reference network is filtered by DNA methylation, then chromatin accessibility of regulatory sites and third by histone marks. TF-gene and miRNA-gene networks are then filtered according to RNA-seq expression of the regulators and edges in the resulting networks are then scored accordingly to the number of filters past and provided as edge weights in the context-specific GRN. 


\subsection{Validation}

To assess the reliability of GRNs generated with Fly T-WEoN, we used as gold-standard a network created with all TF ChIP-seq experiments available in the ENCODE repository for the third instar larval stage or L3 of D. melanogaster. We chose this stage because there are ChIP-seq experiments for 32 different TF and for 10 histone marks as well as RNA-seq data. All experiments considered were carried out in equivalent conditions (see Supp. File NetsInfo for the list and IDs of experiments used). The gold-standard network was created by first removing edges from the reference network arising from genes coding for any regulator that is not among those $32 \mathrm{TFs}$, and second, by removing those edges whose TFBS was not occupied by its respective TF

\subsubsection{Network reliability: edges}

We estimated the performance of Fly T-WEoN by considering the presence/absence of edges in the final network as a binary classification problem. In this set-up, a True Positive (TP) is defined as an edge present in the context-specific network generated after applying the filters and in the gold-standard network. Similarly, a False Negative (FN) edge is absent in the network generated by Fly T-WEoN but it is present in the gold-standard, while a False Positive (FP) edge is present in the network and absent in the gold-standard. Importantly, True Negatives (TNs) indicate edges absent in both the gold-standard and in the network created by Fly T-WEoN. Finally, once all edges are assigned to either of the three types TP, FP or FN, they were used to calculate Precision (P, eq. ), Recall (R, eq. ), and F1 (eq. ), metrics that serve as indicators of the reliability of the context-specific networks. Each of these metrics has a value in the $[0,1]$ range, with greater values indicating a better classification. To evaluate the effect of distance threshold we also calculated the performance metrics using the reference networks generated using the three distance thresholds 1.5, 2 and $5 \mathrm{~Kb}$ (See Supp. file NetsInfo).

$$
\begin{gathered}
P=\frac{T P}{T P+F P} \\
R=\frac{T P}{T P+F N} \\
F 1=\frac{2 P R}{P+R}
\end{gathered}
$$

\subsubsection{Network reliability: local topology}

GRNs are formed by combinations of graphlets, induced subgraphs that have been associated to specific functions [29]. Graphlets can be used to describe local topology of nodes in GRNs, and the presence or absence of the graphlets in which a node participate indicate functional variation for that gene in two realizations of the same network [27]. In addition, the presence or absence of graphlets in two versions of the same network can be considered as a binary classification problem, and thus, the same metrics calculated for edges indicate how similar is the local topology of each gene in the gold-standard network and in the predicted GRNs, or their overall topological similarity. We employed LoTo [26] to calculate precision, recall and the F1 metrics calculated for the presence/absence of graphlets in every pairwise network comparison. If these metrics only consider graphlets in which the same gene participates, they serve to indicate variations in the local topology of that node. Whereas, if the metrics are calculated for all graphlets in the networks, they serve to indicate global topological similarity between the two networks.

\subsection{Fruit fly early embryo development}

To demonstrate the utility of Fly T-WEoN, we generated networks for six different stages of early embryo development in fruit fly (D. melanogaster). We employed RNA-seq experiments and histone marks data downloaded from different databases such as modENCODE and modMine projects [9, 13], and the FlyBase database [18] (see Supp. file NetsInfo for a detailed description of the data used). We downloaded the annotation of the $D$. melanogaster reference genome version 6.32 to process all sequencing experiments. Experiments already mapped to a different version of the reference genome were re-processed or converted using the FlyBase Sequence Coordinates Converter [18]. We employed these data to create context-specific networks for different time points of early development of D. melanogaster. The default 


\begin{tabular}{ccc}
\hline Threshold $(\mathrm{Kb})$ & Genes & Edges \\
\hline 1.5 & 10096 & 82919 \\
2 & 10620 & 89880 \\
5 & 12822 & 127222 \\
\hline
\end{tabular}

Table 2: Gold standard Networks used to validate Fly T-WEoN. Networks made with the 32 TFs at different distance thresholds between TFBSs and the TSS of each gene. Number of different genes and edges present in each of the networks made by assigning a TF to the regulation of a gene if the TF is bound within the distance and the gene TSS.

$1.5 \mathrm{~kb}$ reference network that is included in Fly T-WEoN was used for this example. This reference network comprises 15576 genes (87\% of the total annotated genes of D. melanogaster). Six time-specific networks were created with Fly T-WEoN encompassing the fly embryonic development (0-24h) in time steps of 4 hours (0-4h, 4-8h, 8-12h, 12-16h, 16-20h, and 20-24h), using the available data of histone modifications and RNA-seq.

Finally, we compared each of these networks with the network created for the consecutive time interval using LoTo [26] to calculate overall network similarity and to identify genes whose local topology changed during embryo development according to the F1 calculated for all graphlets in which they participate. For each comparison, we separated nodes by their type (TFs, non-TF protein coding genes and non-coding genes) into four F1 intervals [0-0.5), [0.5-0.7), [0.7-0.9) and [0.9-1.0). For those coding genes that are not TFs in each of these intervals we determined the statistical over-representation of GO-Slim Molecular Process terms with PANTHER using the Fisher's exact test with the Bonferroni correction [28].

\section{Results}

\subsection{Method validation}

We employed the L3 context-specific GRN described in the Methods section to estimate the reliability of the networks generated by our approach. The gold-standard network was made with 32 different TFs and their binding sites determined by ChIP-seq experiments in equivalent experimental conditions. The reference networks made at 1.5, 2 and $5 \mathrm{~kb}$ thresholds are described in table 2. Not surprisingly, larger distance thresholds include more TF-gene interactions for genes to which we can not assign regulators otherwise, and thus, networks built using greater thresholds contain more nodes.

\subsubsection{Network similarity: edges}

Using the L3 example, the lowest score of edges in the predicted networks is two and the highest eleven. This is due to the number of Fly T-WEoN filtering steps applied, so a score of two implies that the TF from which an edge is originated is expressed and there is at least a single histone modification supporting its existence. Scores of three, and above, mean that there is at least two types of histone modification indicatingthat the link exists.

As shown in Table 3 for a threshold of $1.5 \mathrm{~kb}$, Fly T-WEoN generates networks with very high similarity to the gold-standard network in our benchmarking. Starting with edges of score two or greater, the network generated by Fly T-WEoN contains $97.8 \%$ of the edges of the gold-standard network $(\mathrm{R}=$ 0.978), decreasing the recall as the edges score increases. Also, the F1 value follows the same trend, it displays its highest value using this score $(\mathrm{F} 1=0.884)$ and decreases as the minimum score for the edges increases. Moreover, the precision follows a different tendency, with its highest value with score $\geq 6(\mathrm{P}=0.810)$. The worst performance is obtained with a score of eleven, the maximum, with which Fly T-WEoN recovers $0.1 \%$ of the edges of the gold-standard network $(\mathrm{R}=0.001, \mathrm{P}=0.646$ and $\mathrm{F} 1=$ $0.001)$, indicating low similarity between edges present in the predicted networks and the gold-standard.

\subsubsection{Global topological similarity calculated with graphlets}

The trend for graphlet based results is similar to that based on single edges, shown in Table 4 . Using a minimum score of two, Fly T-WEoN is able to recover $95.7 \%$ of the graphlets found in the Gold Standard Network $(\mathrm{R}=0.957)$, but it tends to over predict graphlets as indicated by the much lower precision (P 


\begin{tabular}{ccccccc}
\hline Score & TP & FP & FN & R & P & F1 \\
\hline 2 & 81094 & 19475 & 1825 & $\mathbf{0 . 9 7 8}$ & 0.806 & $\mathbf{0 . 8 8 4}$ \\
3 & 78807 & 18846 & 4112 & 0.950 & 0.807 & 0.873 \\
4 & 76017 & 17998 & 6902 & 0.917 & 0.809 & 0.859 \\
5 & 72802 & 17109 & 10117 & 0.878 & 0.810 & 0.843 \\
6 & 68848 & 16147 & 14071 & 0.830 & $\mathbf{0 . 8 1 0}$ & 0.820 \\
7 & 61477 & 14874 & 21442 & 0.741 & 0.805 & 0.772 \\
8 & 50071 & 12908 & 32848 & 0.604 & 0.795 & 0.686 \\
9 & 5666 & 2225 & 77253 & 0.068 & 0.718 & 0.125 \\
10 & 1512 & 664 & 81407 & 0.018 & 0.695 & 0.036 \\
11 & 62 & 34 & 82857 & 0.001 & 0.646 & 0.001 \\
\hline
\end{tabular}

Table 3: Reliability of L3 gene regulatory networks: single edges. Performance of Fly T-WEoN measured by its ability to recover edges present in the gold-standard network for different scores. The table displays the number of True Positive edges (TP), edges in the gold-standard network also present in the predicted network; False positive edges (FP) or present in the predicted network but absent in the gold-standard network; and False Negative edges, those edges that are are only present in the goldstandard network and are not present in the predicted network. TP, FP and FN edges were used to calculate Precision (P), Recall (R), and F1 (bold numbers indicate their highest values).

\begin{tabular}{ccccccc}
\hline Score & TP & FP & FN & R & P & F1 \\
\hline 2 & 143177569 & 73274608 & 6516537 & $\mathbf{0 . 9 5 7}$ & 0.662 & $\mathbf{0 . 7 8 2}$ \\
3 & 135281974 & 69127626 & 14412132 & 0.904 & 0.662 & 0.764 \\
4 & 125830206 & 63634265 & 23863900 & 0.841 & 0.664 & 0.742 \\
5 & 115565165 & 58106890 & 34128941 & 0.772 & $\mathbf{0 . 6 6 5}$ & 0.715 \\
6 & 103941870 & 52479975 & 45752236 & 0.694 & 0.665 & 0.679 \\
7 & 84304099 & 45038593 & 65390007 & 0.563 & 0.652 & 0.604 \\
8 & 57733273 & 34839940 & 91960833 & 0.386 & 0.624 & 0.477 \\
9 & 757834 & 1231084 & 148936272 & 0.005 & 0.381 & 0.01 \\
10 & 59937 & 123182 & 149634169 & 0 & 0.327 & 0 \\
11 & 229 & 328 & 149693877 & 0 & 0.411 & 0 \\
\hline
\end{tabular}

Table 4: Reliability of L3 gene regulatory networks: graphlets. Performance of Fly T-WEoN measured by its ability to recover graphlets present in the gold-standard network for different edge scores. The table displays the number of True Positive graphlets (TP), graphlets present in the gold-standard network also found in the predicted network; False positive graphlets (FP), present in the predicted network but absent in the gold-standard network; and False Negative graphlets, those that are are only present in the gold-standard network and were not present in the predicted network. TP, FP and FN graphlets were used to calculate Precision (P), Recall (R), and F1 (bold numbers indicate their highest values).

$=0.662)$. Also, the $\mathrm{F} 1$ value had its greatest value with a score of at least two $(\mathrm{F} 1=0.782)$, indicating again high similarity between the predicted and gold-standard networks. The highest value of precision was obtained using at minimum score of five $(\mathrm{P}=0.665)$, this indicating again that networks obtained by Fly T-WEoN contain more graphlets than gold-standard networks, even at the maximum precision. The lowest values for the performance metrics were obtained using weights $\geq 10$, with predicted networks recovering $0 \%$ of the graphlets present in the gold-standard network.

\subsection{An example case: Fruit fly early development}

\subsubsection{Network sizes}

Six time-specific networks were created with Fly T-WEoN encompassing the fly embryonic development (0-24h) in consecutive time ranges of 4 hours (0-4h, 4-8h, 8-12h, 12-16h, 16-20h, and 20-24h). These networks were made using available data of histone modifications and RNA-seq. These networks have different numbers of edges, graphlets, regulatory nodes (TFs) and total number of genes, as shown in Table 5. The largest network belongs to the 16-20h time range, with the largest numbers for nodes, total 


\begin{tabular}{ccccccc}
\hline Graphlet type & $\mathbf{0 - 4 h}$ & $\mathbf{4 - 8 h}$ & $\mathbf{8 - 1 2 h}$ & $\mathbf{1 2 - 1 6 h}$ & $\mathbf{1 6 - 2 0 h}$ & $\mathbf{2 0 - 2 4 h}$ \\
\hline Total edges & 718583 & 733863 & 803613 & 888537 & 928599 & 840567 \\
TF nodes & 305 & 324 & 340 & 335 & 345 & 339 \\
Total nodes & 8811 & 7886 & 8554 & 10528 & 10993 & 11146 \\
\hline
\end{tabular}

Table 5: Characterization of embryo development networks. The table shows the number of edges and regulatory nodes for each of the networks created for the 6 time intervals during early development of the fruit fly. Regulatory nodes indicate the number of TFs in each network and the total number of genes and edges in the networks are also displayed. These networks were obtained by removing unlikely edges from a reference networks were TFBSs located at most at $1.5 \mathrm{~kb}$ upstream the TSS are used to assign the TFs that bind to that TFBS to the regulation of each gene.

\begin{tabular}{ccccccc}
\hline Comparison & TP & FP & FN & R & P & F1 \\
\hline 0-4h $-4-8 \mathrm{~h}$ & 960856575 & 154840150 & 170821683 & 0.849 & 0.861 & 0.855 \\
4-8h - 8-12h & 1015103337 & 237272519 & 100593375 & 0.910 & 0.811 & 0.857 \\
8-12h - 12-16h & 1162138649 & 358797508 & 90237194 & 0.928 & 0.764 & 0.838 \\
12-16h - 16-20h & 1347047992 & 264431780 & 173888152 & 0.886 & 0.836 & 0.860 \\
16-20h - 20-24h & 1297280051 & 74185782 & 314199708 & 0.805 & 0.946 & 0.870
\end{tabular}

Table 6: Comparisons of embryo development networks using gaphlets. The table displays the number of True Positive graphlets (TP), graphlets in the first network (that belonging to the earlier time interval) that are present in the later network; False positive graphlets (FP) are those present in the later network but absent in the earlier one; and False Negative graphlets (FN), those that are are only present in the earlier network and not in the later network. TP, FP and FN graphlets were used to calculate Precision (P), Recall (R), and F1 metrics.

connections, and regulatory nodes (10993, 928599, and 345 respectively). The smallest network is the network for the 0 - $4 \mathrm{~h}$ time range, which has the lowest number of total connections, and regulatory nodes (718583 and 305 respectively), while the network for time range 4 - $8 \mathrm{~h}$ has the lowest number of nodes (7886).

\subsubsection{Network Comparisons}

We compared each network with the network representing the next time interval obtaining F1 values greater than 0.85 (Table 6). These results indicate that despite changes, most of the regulatory network remains unaltered between time lapses. Thus, indicating that relatively small changes in the network account for all stages of early embryo development.

We also analyzed the F1 values by types of genes, TF and non-TF coding, and non-coding genes (Table 7). Without considering gene type (all genes), most of them are in the F1 ranges with less topological variation $([0.7,0.9)$ and $[0.9,1.0])$, evidencing that, as happened with global topology, the local topology of a majority of genes remains unaltered between consecutive time lapses. The same trend is displayed by the TF-coding genes, with most of them in the range $[0.7,0.9)$. With respect to non-TF coding genes, again most of them fall into F1 intervals ranges with less topological variation $([0.7,0.9)$ and $[0.9,1.0])$. Notably, there are large proportions of ncRNA coding genes in the range that displays larger topological variations, hinting they play a relevant role in the developmental stages depicted by the networks. Detailed information on which genes show greater variation on their local topology and the GRN for each time point can be found in the Supp. Material of the article (file LoTo_Embryo and EmbryoNetworks respectively).

\subsubsection{Functional analysis of genes with altered local topology}

After performing comparisons of networks representing consecutive developmental stages, we analyzed the function of genes with altered local topology. To do so, we employed the statistical enrichment of GO-Slim Biological Process terms with PANTHER [38] for genes in each of F1 ranges previously defined. Focusing on the analysis of genes with F1 in the [0-0.5), the enrichment test denoted several GO terms that are known to be involved in embryonic development (Table 8). For example, we found enriched GO terms "developmental process" and "anatomical structure development" in genes in the 


\begin{tabular}{|c|c|c|c|c|c|c|}
\hline & Comparison & $0-4 \mathrm{~h}-4-8 \mathrm{~h}$ & $4-8 \mathrm{~h}-8-12 \mathrm{~h}$ & $8-12 \mathrm{~h}-12-16 \mathrm{~h}$ & $12-16 \mathrm{~h}-16-20 \mathrm{~h}$ & $16-20 \mathrm{~h}-20-24 \mathrm{~h}$ \\
\hline \multirow[t]{4}{*}{ All genes } & {$[0.0,0.5)$} & 1459 & 1398 & 2595 & 2962 & 2511 \\
\hline & {$[0.5,0.7)$} & 187 & 178 & 215 & 319 & 397 \\
\hline & {$[0.7,0.9)$} & 3192 & 1732 & 2023 & 1766 & 1546 \\
\hline & {$[0.9,1.0]$} & 4097 & 5445 & 5820 & 6857 & 7513 \\
\hline \multirow[t]{4}{*}{ TFs } & {$[0.0,0.5)$} & 27 & 25 & 10 & 5 & 10 \\
\hline & {$[0.5,0.7)$} & 7 & 9 & 12 & 7 & 4 \\
\hline & {$[0.7,0.9)$} & 212 & 260 & 302 & 273 & 265 \\
\hline & {$[0.9,1.0]$} & 70 & 47 & 16 & 53 & 66 \\
\hline \multirow[t]{4}{*}{ Coding genes } & {$[0.0,0.5)$} & 953 & 922 & 1787 & 2114 & 1802 \\
\hline & {$[0.5,0.7)$} & 136 & 136 & 151 & 236 & 292 \\
\hline & {$[0.7,0.9)$} & 2602 & 1242 & 1382 & 1126 & 973 \\
\hline & {$[0.9,1.0]$} & 3598 & 4815 & 5077 & 5925 & 6527 \\
\hline \multirow[t]{4}{*}{ Non-coding genes } & {$[0.0,0.5)$} & 479 & 451 & 798 & 843 & 699 \\
\hline & {$[0.5,0.7)$} & 42 & 33 & 52 & 76 & 101 \\
\hline & {$[0.7,0.9)$} & 378 & 230 & 339 & 367 & 308 \\
\hline & {$[0.9,1.0]$} & 429 & 583 & 727 & 879 & 920 \\
\hline
\end{tabular}

Table 7: Total number of genes by type and F1 interval in each of the comparisons of embryo development consecutive networks using gaphlets. The table displays the number of genes in each of the four F1 intervals, $[0.0,0.5),[0.5,0.7),[0.7,0.9)$ and $[0.9,1.0]$ in each of the five comparisons performed between GRNs depicting gene regulation at each time lapse. F1 one values closer to 0 indicate larger local topological variation, while closer to 1 indicate fewer variations in the graphlets in which a gene participates.

lowest F1 range in the comparisons spanning the first 12 hours $(0-4 \mathrm{~h}-4-8 \mathrm{~h}$ and $4-8 \mathrm{~h}-8-12 \mathrm{~h})$. In the comparisons spanning the last 12 hours, we found enriched functional terms related to metabolism and metabolite transport processes such as "glutathione metabolic process", "transmembrane transport", and "aminoglycan metabolic process". Genes in intervals with moderate topological variation (F1 range [0.50.7), see Supp Material GO file) showed enrichment in GO terms related to defense response, metabolic, and developmental process. For the comparison of $4-8 \mathrm{~h}$ and $8-12 \mathrm{~h}$ networks, genes in this F1 range, enriched terms were "animal organ development", "cytoplasmic translation", and "cell development". In the case of the comparison 8-12h-12-16h, enriched terms associated to cell signaling, GO terms "signaling" and "cell communication". Finally, for the comparison of 12-16h-16-20h, overrepresented terms were related with cell structure and cell cycle, GO Slim terms such as "establishment of spindle orientation" and "cell cycle". In the case of the comparison 16-20h - 20-24 no GO term was significantly enriched.

\subsubsection{Subnetworks of nodes showing largest topological variations at early stages}

To further investigate the application of our approach to the early embryo development example, we created subnetworks made of only those nodes that have F1 in the $[0.0,0.5)$ range for each comparison. We then compared subnetworks depicting consecutive stages using LoTo. As an example we show the comparison of the two earlier stages $(0-4 \mathrm{~h}-4-8 \mathrm{~h})$ in figures 2 and 3 the results of the comparison showing only TFs. All these subnetworks can be found as a Cytoscape session in the Supp. Material of the article.

\section{Discussion}

Inference of gene regulation relationships from genomic data is a particularly hard and costly task. This is due to the use of high quality antibodies to determine the bound state of TFs to the open chromatin. Even with aid of computational tools, the determination of gene regulations is an open problem contributed by a gap knowledge of how TFs and other regulators of gene expression work, and by a general lack of genomic data suitable for the prediction of such regulations. The inexpensive RNA-seq and chromatin accessibility through footprint sequencing are commonly used to infer condition-specific networks, but these still require corroboration that again, is usually made with comparisons to ChIP-seq experiments of each TF. However, TF ChIP-seq experiments are unavailable for most conditions of model organisms, including even those that have been deeply studied. Importantly, the number of ChIP-seq used to determine histone modifications are increasing in data repositories, and given the relationship between histone marks and TF binding in chromatin, we created Fly T-WEoN to generate context-specific GRNs in D. melanogaster. 
bioRxiv preprint doi: https://doi.org/10.1101/2020.08.03.232959; this version posted August 4, 2020. The copyright holder for this preprint (which was not certified by peer review) is the author/funder. All rights reserved. No reuse allowed without permission.

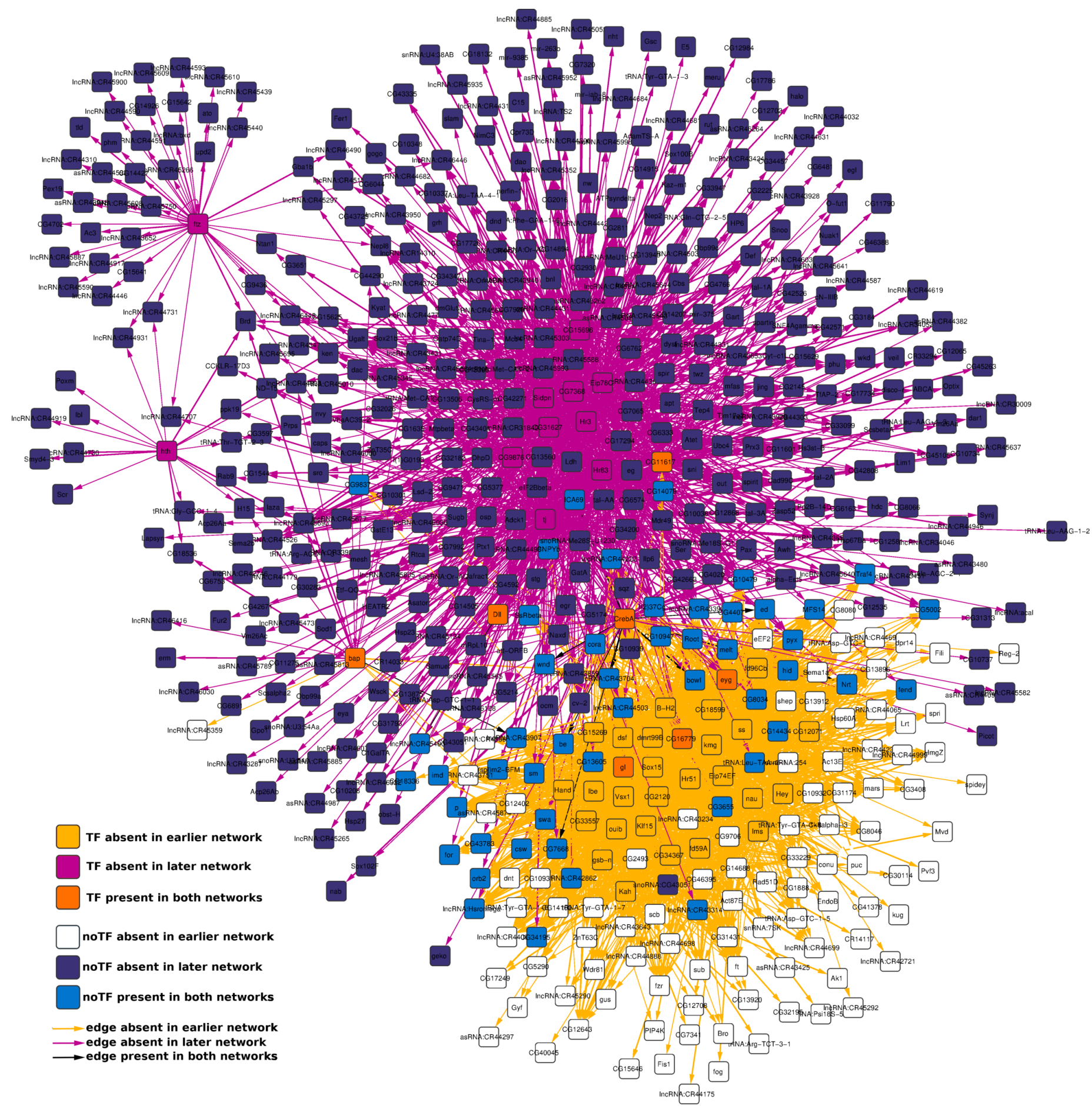

Figure 2: Comparison of subnetworks composed by all those genes showing larger local variation in the $\mathbf{0 - 4 h}-\mathbf{4 - 8 h}$ comparison. The network shown is formed by 594 nodes (44 TFs) and 3107 edges colored accordingly to their existence in the earlier network, in the later network or in both. 


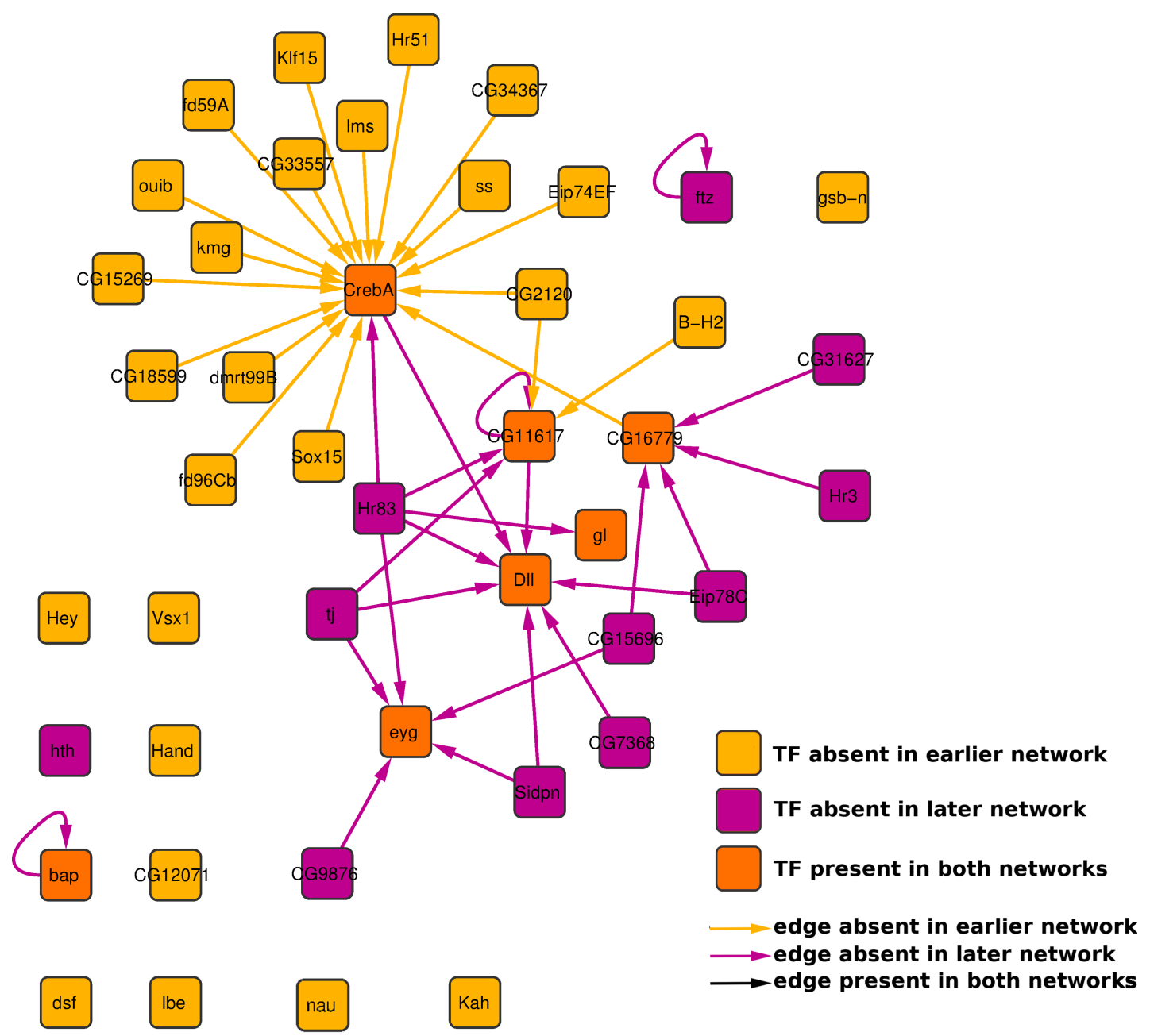

Figure 3: Comparison of subnetworks composed by TF coding genes showing larger local variation in the $\mathbf{0 - 4 h}-\mathbf{4 - 8 h}$ comparison. The network shown is formed by 44 TFs and 42 edges colored accordingly to their existence in the earlier network, in the later network or in both. 


\begin{tabular}{|c|c|c|c|c|}
\hline Comparison & GO Term & GO ID & Fold Enrichment & p-value \\
\hline \multirow{11}{*}{$0-4 h-4-8 h$} & cell differentiation & GO:0030154 & 2.29 & $1.14 \mathrm{e}-04$ \\
\hline & developmental process & GO:0032502 & 2.02 & $1.49 \mathrm{e}-02$ \\
\hline & cellular developmental process & GO:0048869 & 2.15 & $3.12 \mathrm{e}-04$ \\
\hline & sulfur compound metabolic process & GO:0006790 & 2.53 & $1.06 \mathrm{e}-03$ \\
\hline & anatomical structure development & GO:0048856 & 1.92 & $1.41 \mathrm{e}-03$ \\
\hline & cellular modified amino acid metabolic process & GO:0006575 & 2.94 & $1.85 \mathrm{e}-03$ \\
\hline & glutathione metabolic process & GO:0006749 & 3.52 & $2.40 \mathrm{e}-03$ \\
\hline & cell fate commitment & GO:0045165 & 4.82 & $3.54 \mathrm{e}-03$ \\
\hline & neurogenesis & GO:0022008 & 2.38 & $4.14 \mathrm{e}-03$ \\
\hline & generation of neurons & GO:0048699 & 2.38 & $5.81 \mathrm{e}-03$ \\
\hline & multicellular organismal process & GO:0032501 & 1.58 & $9.06 \mathrm{e}-03$ \\
\hline \multirow[t]{5}{*}{$4-8 h-8-12 h$} & developmental process & GO:0032502 & 1.87 & $1.15 \mathrm{e}-03$ \\
\hline & cell differentiation & GO:0030154 & 2.04 & $2.07 \mathrm{e}-03$ \\
\hline & chaperone-mediated protein folding & GO:0061077 & 4.18 & $3.16 \mathrm{e}-03$ \\
\hline & cellular developmental process & GO:0048869 & 1.91 & $4.46 \mathrm{e}-03$ \\
\hline & anatomical structure development & GO:0048856 & 1.79 & $6.33 \mathrm{e}-03$ \\
\hline \multirow[t]{14}{*}{$8-12 h-12-16 h$} & cellular modified amino acid metabolic process & GO:0006575 & 4.18 & $2.61 \mathrm{e}-09$ \\
\hline & glutathione metabolic process & GO:0006749 & 5.00 & $2.04 \mathrm{e}-08$ \\
\hline & cofactor metabolic process & GO:0051186 & 2.24 & $3.25 \mathrm{e}-05$ \\
\hline & sulfur compound metabolic process & GO:0006790 & 2.32 & $1.10 \mathrm{e}-04$ \\
\hline & response to drug & GO:0042493 & 2.92 & $1.39 \mathrm{e}-03$ \\
\hline & organic acid metabolic process & GO:0006082 & 1.66 & $2.81 \mathrm{e}-03$ \\
\hline & small molecule metabolic process & GO:0044281 & 1.46 & $3.85 \mathrm{e}-03$ \\
\hline & transmembrane transport & GO:0055085 & 1.55 & $6.24 \mathrm{e}-03$ \\
\hline & carboxylic acid metabolic process & GO:0019752 & 1.62 & $6.36 \mathrm{e}-03$ \\
\hline & organic anion transport & GO:0015711 & 2.05 & $6.50 \mathrm{e}-03$ \\
\hline & oxoacid metabolic process & GO:0043436 & 1.60 & $6.65 \mathrm{e}-03$ \\
\hline & aminoglycan metabolic process & GO:0006022 & 3.43 & $6.94 \mathrm{e}-03$ \\
\hline & anion transport & GO:0006820 & 1.84 & $8.37 \mathrm{e}-03$ \\
\hline & defense response & GO:0006952 & 3.25 & $8.89 \mathrm{e}-03$ \\
\hline \multirow[t]{8}{*}{ 12-16h - 16-20h } & aminoglycan metabolic process & GO:0006022 & 3.98 & $7.78 \mathrm{e}-04$ \\
\hline & amino sugar catabolic process & GO:0046348 & 3.62 & $2.28 \mathrm{e}-03$ \\
\hline & chemical synaptic transmission & GO:0007268 & 2.09 & $2.73 \mathrm{e}-03$ \\
\hline & anterograde trans-synaptic signaling & GO:0098916 & 2.09 & $2.73 \mathrm{e}-03$ \\
\hline & response to drug & GO:0042493 & 2.64 & $3.05 \mathrm{e}-03$ \\
\hline & synaptic signaling & GO:0099536 & 2.06 & $4.36 \mathrm{e}-03$ \\
\hline & trans-synaptic signaling & GO:0099537 & 2.06 & $4.36 \mathrm{e}-03$ \\
\hline & multicellular organismal process & GO:0032501 & 1.41 & $7.76 \mathrm{e}-03$ \\
\hline \multirow[t]{3}{*}{ 16-20h - 20-24h } & aminoglycan metabolic process & GO:0006022 & 4.25 & $7.65 \mathrm{e}-04$ \\
\hline & amino sugar catabolic process & GO:0046348 & 4.25 & $7.65 \mathrm{e}-04$ \\
\hline & drug metabolic process & GO:0017144 & 1.85 & $5.51 \mathrm{e}-03$ \\
\hline
\end{tabular}

Table 8: GO Slim Biological Process terms associated to genes with the largest topological variation. The table displays the GO Slim Biological Process obtained with PANTHER for genes with $\mathrm{F} 1$ values in the range $[0.0-0.5)$. The Fold Enrichment value indicate the rate between the percentage of genes with the annotation and the percentage of genes with the same annotation in whole genome. If it is greater than 1, it indicates that the category is overrepresented in the data. These results were filtered by a p-value threshold of 0.01 .

With the proposed methodology, we built condition-specific GRNs for the L3 developmental stage and used them to validate our methodology based on the concatenation of simple filters. We employed ChIP-seq for ten different histone marks and 32 different TFs to build gold-standard networks to then compare them Fly T-WEoN networks. Each of the filters in our tool uses current knowledge on the known relationship that exists between epigenetic marks and TF activity. We observed that even if the filtering approach may seem to be too simple it still recovers correctly most of the edges found in the Gold Standard Network we made for that stage. Furthermore, Fly T-WEoN applies a weight system on edges, increasing these weights accordingly to how many filters did each edge past. Our results show that, at least in our test, using a weight of $\geq 2$ produces the most reliable GRNs. This weight means that at least one histone mark and the expression of the TF agrees with the existence of each edge. The worse performance shown with greater weights can be explained due to that by increasing the weight value, the 


\begin{tabular}{|c|c|c|c|c|c|}
\hline Tool & Language & Purpose & Input Data & (Dis) Advantages & GUI \\
\hline $\begin{array}{l}\text { CENTIPEDE } \\
{[31]}\end{array}$ & $\mathrm{R}$ & $\begin{array}{l}\text { Infers bound TFBS from } \\
\text { Chip-seq of histone modifi- } \\
\text { cations and DNAse-seq }\end{array}$ & $\begin{array}{l}\text { Matrix of read counts around mo- } \\
\text { tif matches based on DNAse-seq or } \\
\text { ChIP reads and the follow prior in- } \\
\text { formation: PWM score for motif } \\
\text { matches represented in the genome, } \\
\text { conservation score based on evolu- } \\
\text { tionary information of motif and } \\
\text { motif distance to TSS }\end{array}$ & $\begin{array}{l}\text { Easy to run and very intuitive to } \\
\text { generate output data, however it } \\
\text { needs many previous steps of data } \\
\text { preprocessing to generate the cor- } \\
\text { rect input file. }\end{array}$ & $\overline{\text { no }}$ \\
\hline Anchor [23] & Python & $\begin{array}{l}\text { Predicts in vivo } \mathrm{TF} \text { bindings } \\
\text { profiles across cell types }\end{array}$ & $\begin{array}{l}\text { Genomic coordinates (bed file), } \\
\text { DNase-seq data (BAM file and Big- } \\
\text { Wig file), DNA sequence (genome } \\
\text { fasta file), TFs motifs and Gencode } \\
\text { GFF file }\end{array}$ & $\begin{array}{l}\text { Needs various preprocessing steps } \\
\text { of all data (long times, computing } \\
\text { intensive), then, it is easy to run }\end{array}$ & no \\
\hline Tepic [34] & $\mathrm{C}++, \mathrm{R}$, Python & $\begin{array}{l}\text { Prediction and analysis of } \\
\text { TFBS from epigenetic data, } \\
\text { supporting more than } 30 \\
\text { species }\end{array}$ & $\begin{array}{l}\text { Genome sequence in fasta file, } \\
\text { Genome annotation file (GTF) }\end{array}$ & $\begin{array}{l}\text { Easy to run, moreover the output } \\
\text { is no friendly for posterior analysis } \\
\text { and requires post-processing }\end{array}$ & no \\
\hline Mocap [10] & R, Python & $\begin{array}{l}\text { Classification of TFBSs } \\
\text { from integration of chro- } \\
\text { matin accessibility, motif } \\
\text { scores, TF footprints, } \\
\text { CpG/GC content, evolu- } \\
\text { tionary conservation }\end{array}$ & $\begin{array}{l}\text { DNase-Seq or ATAC-Seq counts, } \\
\text { BigWig, motif matrix }\end{array}$ & $\begin{array}{l}\text { Low time consuming, but it } \\
\text { requires high computing perfor- } \\
\text { mance. It is easy to run, but it } \\
\text { is only available for mouse and } \\
\text { human and the output requires } \\
\text { post-processing }\end{array}$ & no \\
\hline $\begin{array}{l}\text { Fly } \\
\text { WEoN }\end{array}$ & Perl, Java & $\begin{array}{l}\text { Apply filters from different } \\
\text { genomic and epigenomic ex- } \\
\text { periments to a reference net- } \\
\text { work in order to generate } \\
\text { context-specific GRNs }\end{array}$ & $\begin{array}{l}\text { BED files from Histone PTMs, } \\
\text { Methylation sequencing, DNA ac- } \\
\text { cessibility sequencing and RNA-seq } \\
\text { file of counts, RPKM, or FPKM }\end{array}$ & $\begin{array}{l}\text { the major advantage is the possi- } \\
\text { bility to generate a context-specific } \\
\text { GRN without further preprocessing } \\
\text { of data in a friendly way. However } \\
\text { it is only implemented for fly }\end{array}$ & yes \\
\hline
\end{tabular}

Table 9: Qualitative comparison of different methods and Fly T-WEoN. The table indicates for each tool the language used in its implementation, its purpose, its advantages and disadvantages and general user-friendliness.

number of edges and graphlets in the networks decrease. However, using only edges with greater weights decreases the reliability of the edges (tables 3 and 4). Which suggests that the known effect of different epigenetic marks is contradictory, and thus, our simple filtering approach fails to gather the complexity of the epigenetic code.

To highlight the differences and similarities between Fly T-WEoN and other approaches, we report a brief comparison between Fly T-WEoN and other four methods in Table 9. The other methods used for the comparison were CENTIPEDE [31], Anchor [23], TEPIC [34], and Mocap [10]. It is important to stress that none of these methods was designed or even tested for D. melanogaster, and thus a quantitative comparison is not straightforward. Given the heterogeneous data employed by these methods, the absence of actual context-specific GRNs, and the lack of specific tools for D. melanogaster, it is not possible to perform quantitative comparisons between them, and thus, only qualitative comparisons are possible. Our comparison (see Table 9) highlights the main characteristics of Fly T-WEoN, i.e., the intuitive way to use Fly T-WEoN and the integration of its results in Cytoscape, when compared with the other four approaches. It is very important to highlight that these tools use different types of data (see Table 9) in dissimilar context to those used by Fly T-WEoN. This makes even more difficult to make a quantitative performance comparison between them. Also, there is no-context-specific data available for all data types used by Fly T-WEoN (DNAse, RNAseq, DNA methylation and TFs ChIP-seq), which does not allow for a full comparison.

Regarding the example of embryonic development of D. melanogaster, we created 6 different networks, each depicting transcriptional control by TFs for each of the four hours intervals of the first 24 hour of a fly embryo. We opted for this condition and time intervals because these were the conditions for which there is more epigenetic and transcriptional data at modENCODE and GEO datasets. Importantly, the stages represented by our networks are when cells and tissues in D. melanogaster are more homogeneous, and thus, all omic data employed is deemed to be more significant. When comparing these GRNs with LoTo [26], we observed that the networks increase the number of nodes and connections as development progresses. This may indicate that in later stages of development transcriptional regulation becomes a more complex process that involves a grater number of TFs in greater number of cell fates and tissues. Comparisons of overall similarity between networks representing consecutive time intervals showed that the largest variation takes place between $8-12 \mathrm{~h}$ and $12-16 \mathrm{~h}$ networks and that the overall topology of the networks changes less is in the last transition between developmental stages included, i.e. in the 
comparison 16-20h and 20-24h networks.

With respect to variations in the local topology of single nodes determined by F1 calculated for the presence/absence of graphlets, most genes had small variations in all comparisons 7, a trend observed for all gene types in the networks (TFs, protein coding and ncRNAs). The only exception is ncRNA coding genes, mainly lncRNAs, which are almost as numerous in the F1 range that indicates largest topological variation as in the range depicting the lowest variation. These findings agree with previous knowledge on the role played by lncRNA in D. melanogaster development [24]. Regarding our observation of relatively few TFs displaying large variations in their local topology, and that those with larger changes (lowest F1) are densely linked between them, these findings agree with the concept of clusters of master regulators [14]. In this concept, a small cluster of highly interconnected TFs are the master regulators controlling the other regulators whose function is to act as efectors or "fine-tuners" of the orders given by the master regulators. In our example, regarding the master regulator concept, the "fine-tuners" would regulatory nodes found in the F1 ranges with higher values that are linked to the master regulators and to many other genes that do not code for regulators. Nonetheless, it should also be considered that specially at the earlier stages of embryo, there are many TFs that are inherited from the mother [1], and given that our approach uses as approximation for TF activity the expression of their coding genes, maternal TFs are disregarded. The fact of observing an increasing number of nodes as the networks depict later stages also agrees with known facts regarding developments, as tissues and specialized cells appear, both regulators and non regulator genes tend to perform more specialized functions [1]. Our functional analysis also corroborates this (see Table 8), more general functions related at the earlier stages and more specialized functions as development progresses, validating again the networks generated with Fly T-WEoN.

\section{Conclusion}

Here, we demonstrated the reliability of our tool, Fly T-WEoN, with results indicating that most of the regulatory events depicted by edges in its resulting networks are likely taking place. In addition to this validation, and given the current lack of tools that integrate epigenetic data for the construction of GRNs in D. melanogaster, we also provided a qualitative comparison with other approaches, helping in this way to stress out the usability of our method. The minimum input required by Fly T-WEoN is a quantification of the expression of genes, but the results we show here prove how the quality of the network improves by using other epigenetic data or quantification of miRNAs.

We finally demonstrated through a case study the usefulness of genomic data to filter out known regulations from a reference network and make context-specific gene regulatory networks where functions of genes with varying regulation correlate with the development stage. Moreover, we developed a Cytoscape app for Fly T-WEoN that serves as frontend for the presented method, allowing users to create and visualize context-specific GRNs from their processed RNA-seq, DNase-seq, bisulphite-seq, and ChIP-seq datasets or data obtained from public databases. We expect to further develop a backend software harnessing Machine Learning algorithms that would allow final users to predict gene expression from minimal and cheap genomic data, and extend the current method from fruit fly to other model organisms, specially human.

Fly T-WEoN can be obtained free of charge here https://weon.readthedocs.io. Supp. Material files con be accessed here https://figshare.com/projects/WEoN_FlyT/76983.

\section{Acknowledgements}

We acknowledge the help received from Dr. Inti Pedroso for his patience and useful discussions and Dr Yesid Cuesta for his constructive review of the manuscript.

\section{Funding}

FONDECYT project 1181089 from Agencia Nacional de Investigación Científica y Desarrollo. ANID Ph.D. Fellowship \#21191197 to SC and \#21201856 to LM, and Universidad Mayor Ph.D. scholarships to EM and CV. 


\section{References}

[1] Boris Adryan and Sarah A. Teichmann. The developmental expression dynamics of Drosophila melanogaster transcription factors. Genome Biology, 11(4), apr 2010.

[2] Robin Andersson and Albin Sandelin. Determinants of enhancer and promoter activities of regulatory elements. Nature Reviews Genetics, 21(2):71-87, 2020.

[3] David P. Bartel. MicroRNAs: target recognition and regulatory functions. Cell, 136(2):215-33, jan 2009.

[4] Teresa K. Barth and Axel Imhof. Fast signals and slow marks: the dynamics of histone modifications. Trends Biochem. Sci., 35(11):618-626, 2010.

[5] Eva Bártová, Jana Krejcí, Andrea Harnicarová, Gabriela Galiová, and Stanislav Kozubek. Histone modifications and nuclear architecture: a review. J. Histochem. Cytochem., 56(8):711-21, aug 2008.

[6] Charles Blatti, Majid Kazemian, Scot Wolfe, Michael Brodsky, and Saurabh Sinha. Integrating motif, DNA accessibility and gene expression data to build regulatory maps in an organism. Nucleic Acids Research, 43(8):3998-4012, apr 2015.

[7] Imre M. Boros. Histone modification in drosophila. Brief. Funct. Genomics, 11(4):319-331, 2012.

[8] Richard W. Carthew and Erik J. Sontheimer. Origins and Mechanisms of miRNAs and siRNAs. Cell, 136(4):642-655, feb 2009.

[9] Susan E. Celniker, Laura A. L. Dillon, Mark B. Gerstein, Kristin C. Gunsalus, Steven Henikoff, Gary H. Karpen, Manolis Kellis, Eric C. Lai, Jason D. Lieb, David M. MacAlpine, Gos Micklem, Fabio Piano, Michael Snyder, Lincoln Stein, Kevin P. White, and Robert H. Waterston. Unlocking the secrets of the genome. Nature, 459(7249):927-930, jun 2009.

[10] Xi Chen, Bowen Yu, Nicholas Carriero, Claudio Silva, and Richard Bonneau. Mocap: large-scale inference of transcription factor binding sites from chromatin accessibility. Nucleic Acids Research, 45(8):4315-4329, may 2017.

[11] Chih Hung Chou, Sirjana Shrestha, Chi Dung Yang, Nai Wen Chang, Yu Ling Lin, Kuang Wen Liao, Wei Chi Huang, Ting Hsuan Sun, Siang Jyun Tu, Wei Hsiang Lee, Men Yee Chiew, Chun San Tai, Ting Yen Wei, Tzi Ren Tsai, Hsin Tzu Huang, Chung Yu Wang, Hsin Yi Wu, Shu Yi Ho, Pin Rong Chen, Cheng Hsun Chuang, Pei Jung Hsieh, Yi Shin Wu, Wen Liang Chen, Meng Ju Li, Yu Chun Wu, Xin Yi Huang, Fung Ling Ng, Waradee Buddhakosai, Pei Chun Huang, Kuan Chun Lan, Chia Yen Huang, Shun Long Weng, Yeong Nan Cheng, Chao Liang, Wen Lian Hsu, and Hsien Da Huang. MiRTarBase update 2018: A resource for experimentally validated microRNA-target interactions. Nucleic Acids Research, 46(D1):D296-D302, jan 2018.

[12] Melissa S Cline, Michael Smoot, Ethan Cerami, Allan Kuchinsky, Nerius Landys, Chris Workman, Rowan Christmas, Iliana Avila-Campilo, Michael Creech, Benjamin Gross, Kristina Hanspers, Ruth Isserlin, Ryan Kelley, Sarah Killcoyne, Samad Lotia, Steven Maere, John Morris, Keiichiro Ono, Vuk Pavlovic, Alexander R Pico, Aditya Vailaya, Peng-Liang Wang, Annette Adler, Bruce R Conklin, et al. Integration of biological networks and gene expression data using Cytoscape. Nature Protocols, 2(10):2366-82, jan 2007.

[13] S. Contrino, R. N. Smith, D. Butano, A. Carr, F. Hu, R. Lyne, K. Rutherford, A. Kalderimis, J. Sullivan, S. Carbon, E. T. Kephart, P. Lloyd, E. O. Stinson, N. L. Washington, M. D. Perry, P. Ruzanov, Z. Zha, S. E. Lewis, L. D. Stein, and G. Micklem. modMine: flexible access to modENCODE data. Nucleic Acids Research, 40(D1):D1082-D1088, jan 2012.

[14] Trevor L. Davis and Ilaria Rebay. Master regulators in development: Views from the Drosophila retinal determination and mammalian pluripotency gene networks. Developmental Biology, 421(2):93107, jan 2017.

[15] Guillaume J Filion, Joke G van Bemmel, Ulrich Braunschweig, Wendy Talhout, Jop Kind, Lucas D Ward, Wim Brugman, Inês J de Castro, Ron M Kerkhoven, Harmen J Bussemaker, and Bas van Steensel. Systematic protein location mapping reveals five principal chromatin types in Drosophila cells. Cell, 143(2):212-24, oct 2010. 
[16] Simon Fishilevich, Ron Nudel, Noa Rappaport, Rotem Hadar, Inbar Plaschkes, Tsippi Iny Stein, Naomi Rosen, Asher Kohn, Michal Twik, Marilyn Safran, Doron Lancet, and Dana Cohen. GeneHancer: genome-wide integration of enhancers and target genes in GeneCards. Database, 2017, jan 2017.

[17] Luz Garcia-Alonso, Mahmoud M Ibrahim, Denes Turei, and Julio Saez-Rodriguez. Benchmark and integration of resources for the estimation of human transcription factor activities. bioRxiv, p. 337915 , jun 2018.

[18] L. Sian Gramates, Steven J. Marygold, Gilberto dos Santos, Jose-Maria Urbano, Giulia Antonazzo, Beverley B. Matthews, Alix J. Rey, Christopher J. Tabone, Madeline A. Crosby, David B. Emmert, Kathleen Falls, Joshua L. Goodman, Yanhui Hu, Laura Ponting, Andrew J. Schroeder, Victor B. Strelets, Jim Thurmond, and Pinglei Zhou. FlyBase at 25: looking to the future. Nucleic Acids Research, 45(D1):D663-D671, jan 2017.

[19] Aziz Khan, Oriol Fornes, Arnaud Stigliani, Marius Gheorghe, Jaime A Castro-Mondragon, Robin Van Der Lee, Adrien Bessy, Jeanne Chèneby, Shubhada R Kulkarni, Ge Tan, Damir Baranasic, David J Arenillas, Albin Sandelin, Klaas Vandepoele, Boris Lenhard, Benoît Ballester, Wyeth W Wasserman, François Parcy, and Anthony Mathelier. JASPAR 2018: Update of the open-access database of transcription factor binding profiles and its web framework. Nucleic Acids Research, 46(D1):D260-D266, jan 2018.

[20] Peter V. Kharchenko, Artyom A. Alekseyenko, Yuri B. Schwartz, Aki Minoda, Nicole C. Riddle, Jason Ernst, Peter J. Sabo, Erica Larschan, Andrey A. Gorchakov, Tingting Gu, Daniela LinderBasso, Annette Plachetka, Gregory Shanower, Michael Y. Tolstorukov, Lovelace J. Luquette, Ruibin Xi, Youngsook L. Jung, Richard W. Park, Eric P. Bishop, Theresa K. Canfield, Richard Sandstrom, Robert E. Thurman, David M. MacAlpine, John A. Stamatoyannopoulos, Manolis Kellis, Sarah C.R. Elgin, Mitzi I. Kuroda, Vincenzo Pirrotta, Gary H. Karpen, and Peter J. Park. Comprehensive analysis of the chromatin landscape in Drosophila melanogaster. Nature, 471(7339):480-486, mar 2011.

[21] Ivan Krivega and Ann Dean. Enhancer and promoter interactions-long distance calls. Curr. Opin. Genet. Dev., 22(2):79-85, 2012.

[22] Samuel A Lambert, Arttu Jolma, Laura F Campitelli, Pratyush K Das, Yimeng Yin, Mihai Albu, Xiaoting Chen, Jussi Taipale, Timothy R Hughes, and Matthew T Weirauch. The Human Transcription Factors. Cell, 172(4):650-665, feb 2018.

[23] Hongyang Li, Daniel Quang, and Yuanfang Guan. Anchor: Trans-cell type prediction of transcription factor binding sites. Genome Research, 29(2):281-292, feb 2019.

[24] Keqin Li, Yuanliangzi Tian, Ya Yuan, Xiaolan Fan, Mingyao Yang, Zhi He, and Deying Yang. Insights into the functions of LncRNAs in drosophila. International Journal of Molecular Sciences, 20(18), sep 2019.

[25] Zhi-Ping Liu, Canglin Wu, Hongyu Miao, and Hulin Wu. RegNetwork: an integrated database of transcriptional and post-transcriptional regulatory networks in human and mouse. Database, 2015:bav095, sep 2015.

[26] Alberto J. Martin, Sebastián Sebastian Sebasti?n Contreras-Riquelme, Calixto Dominguez, and Tomas Perez-Acle. LoTo : a graphlet based method for the comparison of local topology between gene regulatory networks. PeerJ, 5:1-22, feb 2017.

[27] Alberto J M Martin, Calixto Dominguez, Sebastián Contreras-Riquelme, David S Holmes, and Tomas Perez-Acle. Graphlet Based Metrics for the Comparison of Gene Regulatory Networks. PLoS One, 11(10):e0163497, jan 2016

[28] Huaiyu Mi, Anushya Muruganujan, John T Casagrande, and Paul D Thomas. Large-scale gene function analysis with the PANTHER classification system. Nature protocols, 8(8):1551-66, aug 2013.

[29] R Milo, S Shen-Orr, S Itzkovitz, N Kashtan, D Chklovskii, and U Alon. Network motifs: simple building blocks of complex networks. Science (80-. )., 298(5594):824-7, oct 2002. 
[30] Luisa Morales-Nebreda, Fred S. McLafferty, and Benjamin D. Singer. DNA methylation as a transcriptional regulator of the immune system. Translational Research, 204:1-18, feb 2019.

[31] Roger Pique-Regi, Jacob F Degner, Athma A Pai, Daniel J Gaffney, Yoav Gilad, and Jonathan K Pritchard. Accurate inference of transcription factor binding from DNA sequence and chromatin accessibility data. Genome Research, 21(3):447-455, mar 2011.

[32] Nicole C. Riddle, Aki Minoda, Peter V. Kharchenko, Artyom A. Alekseyenko, Yuri B. Schwartz, Michael Y. Tolstorukov, Andrey A. Gorchakov, Jacob D. Jaffe, Cameron Kennedy, Daniela LinderBasso, Sally E. Peach, Gregory Shanower, Haiyan Zheng, Mitzi I. Kuroda, Vincenzo Pirrotta, Peter J. Park, Sarah C R Elgin, and Gary H. Karpen. Plasticity in patterns of histone modifications and chromosomal proteins in Drosophila heterochromatin. Genome Research, 21(2):147-163, 2011.

[33] Scott B. Rothbart and Brian D. Strahl. Interpreting the language of histone and DNA modifications, aug 2014.

[34] Florian Schmidt, Nina Gasparoni, Gilles Gasparoni, Kathrin Gianmoena, Cristina Cadenas, Julia K. Polansky, Peter Ebert, Karl Nordström, Matthias Barann, Anupam Sinha, Sebastian Fröhler, Jieyi Xiong, Azim Dehghani Amirabad, Fatemeh Behjati Ardakani, Barbara Hutter, Gideon Zipprich, Bärbel Felder, Jürgen Eils, Benedikt Brors, Wei Chen, Jan G. Hengstler, Alf Hamann, Thomas Lengauer, Philip Rosenstiel, Jörn Walter, and Marcel H. Schulz. Combining transcription factor binding affinities with open-chromatin data for accurate gene expression prediction. Nucleic Acids Research, 45(1):54-66, jan 2017.

[35] Dirk Schübeler, David M. MacAlpine, David Scalzo, Christiane Wirbelauer, Charles Kooperberg, Fred Van Leeuwen, Daniel E. Gottschling, Laura P. O’Neill, Bryan M. Turner, Jeffrey Delrow, Stephen P. Bell, and Mark Groudine. The histone modification pattern of active genes revealed through genome-wide chromatin analysis of a higher eukaryote. Genes Dev., 18(11):1263-1271, jun 2004 .

[36] Marcel H Schulz, William E Devanny, Anthony Gitter, Shan Zhong, Jason Ernst, and Ziv Bar-Joseph. DREM 2.0: Improved reconstruction of dynamic regulatory networks from time-series expression data. BMC Syst. Biol., 6(1):104, aug 2012.

[37] Tiago C Silva, Antonio Colaprico, Catharina Olsen, Fulvio D’Angelo, Gianluca Bontempi, Michele Ceccarelli, and Houtan Noushmehr. TCGA Workflow: Analyze cancer genomics and epigenomics data using Bioconductor packages. F1000Research, 5:1542, 2016.

[38] Paul D. Thomas, Michael J. Campbell, Anish Kejariwal, Huaiyu Mi, Brian Karlak, Robin Daverman, Karen Diemer, Anushya Muruganujan, and Apurva Narechania. PANTHER: A library of protein families and subfamilies indexed by function. Genome Research, 13(9):2129-2141, sep 2003.

[39] Feifei Xiao, Zhixiang Zuo, Guoshuai Cai, Shuli Kang, Xiaolian Gao, and Tongbin Li. miRecords: An integrated resource for microRNA-target interactions. Nucleic Acids Research, 37(D1):D105-10, jan 2009 .

[40] Jingping Yang and Victor G Corces. Insulators, long-range interactions, and genome function. Curr. Opin. Genet. Dev., 22(2):86-92, 2012.

[41] Jiro C. Yasuhara and Barbara T. Wakimoto. Molecular landscape of modified histones in Drosophila heterochromatic genes and euchromatin-heterochromatin transition zones. PLoS Genet., 4(1):0159$0172,2008$.

[42] Hang Yin, Sarah Sweeney, Debasish Raha, Michael Snyder, and Haifan Lin. A High-Resolution Whole-Genome Map of Key Chromatin Modifications in the Adult Drosophila melanogaster. PLoS Genet., 7(12):e1002380, dec 2011. 